Correspondence

Tom S. J. Elliott

Tom.Elliott@uhb.nhs.uk

Received 9 February 2006

Accepted 5 September 2006

\section{Evaluation of routine microbiological techniques for establishing the diagnosis of catheter-related bloodstream infection caused by coagulase- negative staphylococci}

\author{
Anna L. Casey, ${ }^{1}$ Tony Worthington, ${ }^{2}$ Peter A. Lambert ${ }^{2}$ and Tom S. J. Elliott ${ }^{1}$ \\ ${ }^{1}$ Department of Clinical Microbiology and Infection Control, University Hospital Birmingham \\ NHS Foundation Trust, The Queen Elizabeth Hospital, Edgbaston, Birmingham B15 2TH, UK \\ ${ }^{2}$ Department of Pharmaceutical and Biological Sciences, Aston University, Aston Triangle, \\ Birmingham B4 7ET, UK
}

\begin{abstract}
Microbiological diagnosis of catheter-related bloodstream infection (CR-BSI) is often based on isolation of indistinguishable micro-organisms from an explanted catheter tip and blood culture, confirmed by antibiograms. Whether phenotypic identification of coagulase-negative staphylococci (CoNS) allows an accurate diagnosis of CR-BSI to be established was evaluated. Eight patients with a diagnosis of CR-BSI had CoNS isolated from pure blood cultures and explanted catheter tips which were considered as indistinguishable strains by routine microbiological methods. For each patient, an additional three colonies of CoNS isolated from the blood and five from the catheter tip were subcultured and further characterized by antibiogram profiles, analytical profile index (API) biotyping and PFGE. PFGE distinguished more strains of CoNS compared to API biotyping or antibiograms (17, 10 and 11, respectively). By PFGE, indistinguishable micro-organisms were only isolated from pure blood and catheter tip cultures in four out of eight (50\%) patients thus supporting the diagnosis of CR-BSI. In another patient, indistinguishable micro-organisms were identified in both cultures; however, other strains of CoNS were also present. The remaining three patients had multiple strains of CoNS, none of which were indistinguishable in the tip and blood cultures, thus questioning the diagnosis of CR-BSI. Phenotypic characterization of CoNS lacked discriminatory power. Current routine methods of characterizing a limited number of pooled colonies may generate misleading results as multiple strains may be present in the cultures. Multiple colonies should be studied using a rapid genotypic characterization method to confirm or refute the diagnosis of CR-BSI.
\end{abstract}

\section{INTRODUCTION}

The laboratory diagnosis of catheter-related bloodstream infection (CR-BSI) due to coagulase-negative staphylococci (CoNS) by current microbiological methods is often complicated, since CoNS recovered from clinical samples may represent contamination from the skin surface, catheter colonization or actual infection. As CoNS are the most frequent cause of CR-BSI, accurate identification of strains recovered from the catheter tip and blood cultures is essential to establish a correct diagnosis, otherwise patients may be unnecessarily treated with potentially toxic antibiotics and intravascular catheters may be inappropriately removed.

Abbreviations: API, analytical profile index; CoNS, coagulase-negative staphylococci; CR-BSI, catheter-related bloodstream infection.
Microbiological diagnosis of CR-BSI following removal of a catheter is often established by the recovery of indistinguishable micro-organisms from the catheter tip and blood cultures of patients with suspected infection. The identity is usually based on microbial speciation and antibiogram profiles (O'Grady et al., 2002; Worthington \& Elliott, 2005). However, recent reports suggest that cultures of colonially indistinguishable CoNS may indeed contain multiple different strains (Viedma et al., 2000; Kloos \& Bannerman, 1994).

Characterization of CoNS recovered from clinical samples including catheter tips and blood cultures in the routine microbiology laboratory is commonly based on only biochemical profiles and antibiograms. It has been suggested that routine antimicrobial susceptibility testing of CoNS isolated from blood cultures is unnecessary (Chandran \& Rennie, 2005). However, if routine antibiotic susceptibility testing is restricted, antibiograms will not be available to aid 
the diagnosis of CR-BSI. It is therefore important to determine whether the use of antibiograms in conjunction with biochemical identification tests for characterization of CoNS is sufficiently sensitive to distinguish epidemiological relationships. Indeed, recent work in our laboratory has suggested that phenotypically indistinguishable strains of CoNS may be genetically unrelated when characterized by PFGE, which is highly discriminatory (Casey et al., 2005).

In this investigation, we evaluated whether routine identification of CoNS by current phenotypic methods is sufficient to facilitate the accurate diagnosis of CR-BSI and whether multiple strains of CoNS may be associated with CR-BSI.

\section{METHODS}

Patients. Approval was obtained for this study from the Local Research Ethics Committee prior to commencement and informed consent was given by all participants. Eight stem cell transplant patients (five male and three female) in the bone marrow transplant unit, University Hospital Birmingham NHS Foundation Trust, who had a Hickman catheter in situ and a clinical diagnosis of CR-BSI due to CoNS were recruited into the study following informed written consent. The mean patient age was 50 years (range $=18-77$ ). The clinical diagnosis of CR-BSI was based on the presence of two or more of the following: erythema, oedema and purulent exudate around the catheter exit site; low-grade pyrexia with no underlying cause $\left(\leqslant 38.5^{\circ} \mathrm{C}\right)$; rigors post-flushing of the catheter; the pyrexia being unresponsive to broad-spectrum antibiotics; and cessation of symptoms following catheter removal (O'Grady et al., 2002). This diagnosis was confirmed microbiologically by recovery of indistinguishable micro-organisms (based on speciation and antibiogram) from the catheter tip and blood culture obtained from a peripheral vein (O'Grady et al., 2002).

Routine isolation and identification of CoNS. Blood and catheter tip cultures were performed in accordance with routine laboratory standard operating procedures. The distal $5 \mathrm{~cm}$ of the explanted central venous catheter was sampled by rolling the tip over the surface of a $7 \%$ blood agar plate (Maki et al., 1977). Blood cultures were performed using the Bactec 9240 automated system (BD). At least four colonies from morphologically pure cultures of CoNS recovered from blood cultures and catheter tips of each patient were combined and used for routine identification and antibiotic sensitivity testing.

Further characterization of CoNS recovered from blood cultures and catheter tips. For each patient, a further three CoNS colonies from the primary isolation plates of blood culture and similarly five from the catheter tip were individually subcultured onto blood agar to obtain pure cultures for phenotypic and genotypic characterization.

API biotyping. All isolates were biotyped by the API ID 32 STAPH (bioMérieux).

Antibiograms. Eight isolates from each patient were characterized by antibiogram in accordance with the BSAC standardized disc diffusion guidelines (Andrews, 2001). The antibiotics employed were: $1 \mu \mathrm{g}$ oxacillin, $5 \mu \mathrm{g}$ erythromycin, $5 \mu \mathrm{g}$ trimethoprim, $10 \mu \mathrm{g}$ fusidin, $2 \mu \mathrm{g}$ rifampicin, $5 \mu \mathrm{g}$ and $200 \mu \mathrm{g}$ mupirocin, $10 \mu \mathrm{g}$ tetracycline, $10 \mu \mathrm{g}$ gentamicin and $2 \mu \mathrm{g}$ clindamycin.

Molecular characterization of CoNS by PFGE. All isolates were characterized genotypically by SmaI chromosome macrorestriction profiling using PFGE and analysed with the GelCompar computer software as outlined previously (Casey et al., 2005). Criteria for bacterial strain typing by PFGE were employed to distinguish strains (Tenover et al., 1995).

\section{RESULTS AND DISCUSSION}

\section{Comparison of phenotypic and genotypic typing methods}

Analysis of the phenotypic and genotypic characteristics of the 64 isolates of the CoNS (eight from each patient) demonstrated that 17 strains of CoNS were distinguished by PFGE as compared to 10 by API biotyping and 11 by antibiograms. These results concur with those achieved in previous studies demonstrating that genotypic typing techniques are more discriminative than phenotypic typing methods for the characterization of staphylococci (Tenover et al., 1994; Toldos et al., 1997).

\section{The diagnosis of CR-BSI}

The frequency of diagnosis of CR-BSI based on an indistinguishable strain of CoNS being isolated from the blood and catheter tip culture using API biotyping, antibiogram typing and PFGE is presented in Fig. 1. All eight patients had apparently indistinguishable CoNS isolated from the blood culture and catheter tips when compared by antibiogram using at least four combined colonies from each morphologically pure culture. Further phenotypic characterization of the CoNS by API biotyping alone suggested that seven out of eight patients (patients 1, $2,3,5,6,7$ and 8 ) had at least one indistinguishable strain in both samples thus supporting the clinical diagnosis of CRBSI. This observation was confirmed by the antibiogram profiles in the same patients. However, PFGE only demonstrated indistinguishable strains of CoNS in patients $1,2,3,5$ and 6 .

Many cultures of CoNS obtained from patients are considered by routine microbiology laboratories to be pure as there may be no variation in colonial morphology of the CoNS following $24 \mathrm{~h}$ incubation. Consequently, identification of CoNS and antibiotic sensitivity testing is often undertaken on several combined colonies (Andrews, 2001). The results of this study demonstrated that some CoNS cultures identified as being pure by their indistinguishable colonial appearance following $24 \mathrm{~h}$ incubation contained more than one strain of CoNS. Indeed, antibiogram typing demonstrated that in patients 5, 6 and 7 , two strains were present in the catheter tip culture alone and in patient 8 , two strains were present in both cultures. API biotyping demonstrated that there were two strains in the catheter tip culture of patient 6 , two strains in both cultures from patient 7 and three strains in both cultures from patient 8. With PFGE patients 1, 2, 3 and 5 had pure cultures of indistinguishable strains of CoNS present in both the blood and tip cultures. Patient 6 also had indistinguishable strains present but with other dissimilar strains. Two 


\section{Typing technique}

(A) Initial antibiograms carried out on

\section{four combined colonies
(B) Further antibiograms carried out
on multiple colonies \\ four combined colonies
(B) Further antibiograms carried out
on multiple colonies \\ four combined colonies
(B) Further antibiograms carried out
on multiple colonies} (C) API biotyping carried out on
multiple colonies
(D) Pulsed-field gel electrophoresis carried out on multiple colonies

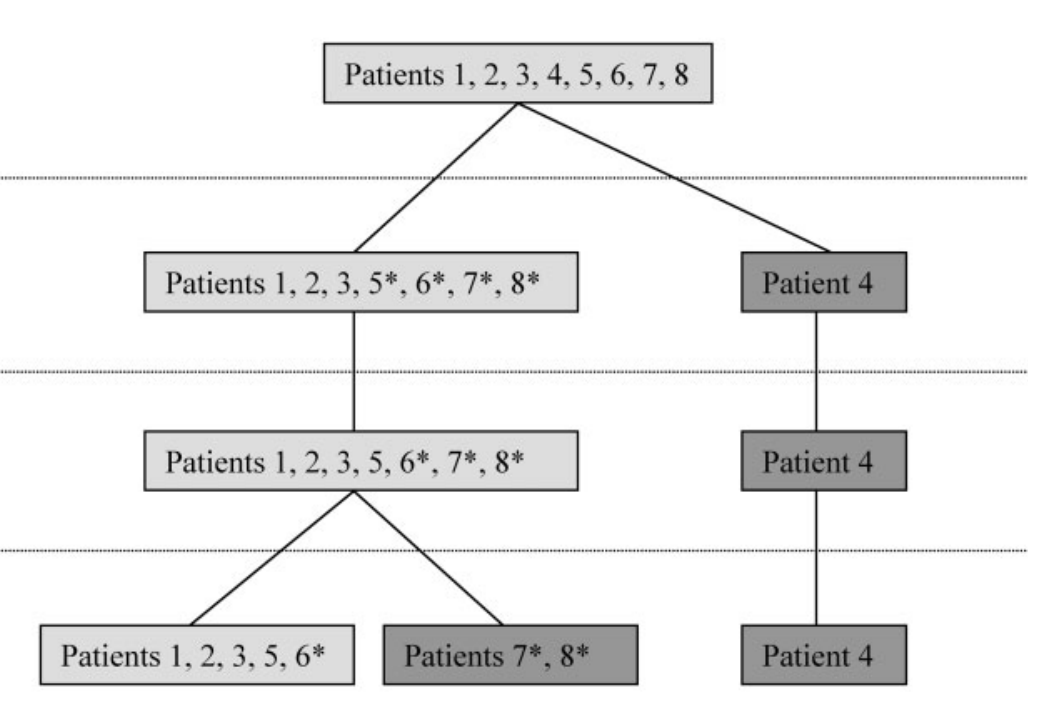

Fig. 1. A flowchart outlining the relatedness of CoNS isolated from blood cultures and catheter tips of eight patients based on different typing techniques (A-D). All patients had apparently indistinguishable CoNS when assessed by the initial antibiogram (A); however, following further antibiotic sensitivity testing (B), patient 4 had dissimilar strains and in patients 5,6 , 7 and 8 multiple strains were present. This pattern was confirmed in patients 6,7 and 8 by API. Using the most discriminative of typing techniques, PFGE (D), only four patients (1, 2, 3 and 5) had similar strains in both blood and tip cultures with the absence of multiple strains. Patient 6 , however, had indistinguishable strains present but with other dissimilar strains. Using antibiogram typing (B), API biotyping (C) and PFGE (D), patient 4 had no indistinguishable isolates from blood and catheter tip culture. Patients 7 and 8 also had no indistinguishable isolates whilst using PFGE. Multiple strains of CoNS were also detected in the blood and/or tip cultures of patients 5, 6, 7 and 8 by at least one typing technique. Light shading, patients whose isolates from blood culture were indistinguishable from those from the catheter tip culture; dark shading, patients in whom no isolates from the blood culture were found to be indistinguishable from any isolate from the catheter tip culture. *Patients in whom multiple strains were present in blood and/or catheter tip culture.

strains of CoNS were present in the catheter tip culture in patient 6, patient 7 had two strains of CoNS present in both cultures and in patient 8 there were two strains in the catheter tip culture and three in the blood culture (Fig. 2). Indeed, it has been documented that a $24 \mathrm{~h}$ incubation period is inadequate to observe differences in colonial morphology in CoNS cultures (Kloos \& Bannerman, 1994). In a recent study, CoNS recovered from catheter tip cultures were morphologically indistinguishable after $24 \mathrm{~h}$ incubation; however, after $72 \mathrm{~h}$ clear differences in colonial morphology were observed. Further studies are required to investigate the optimal incubation period required to observe morphological differences in cultures of CoNS but this may offer a simple approach to distinguish different strains and to identify all morphologically distinct colonial types.

The presence of multiple strains of CoNS on catheter tips and blood cultures may represent contamination of the catheter on removal by skin flora or micro-organisms colonizing the internal component of the luer ports, neither causing the bloodstream infection. It would be of concern if a limited number of isolates were selected for analysis as the CoNS strain causing the CR-BSI may not be selected for identification and characterization including antibiotic sensitivity testing. This may have been the case for patient 4 , where none of the strains isolated from the catheter tip matched those from the blood culture using any of the typing techniques despite routine microbiological tests confirming the diagnosis of CR-BSI (Fig. 3). The original diagnosis was based on an indistinguishable antibiogram achieved from four combined colonies from the primary isolation plates of the blood culture and from four combined colonies obtained from the catheter tip culture of this patient. When using PFGE, patients 7 and 8 also had no indistinguishable CoNS strains in the tip and blood culture (Fig. 2).

When using antibiotic sensitivity patterns for typing, indistinguishable strains were identified in patients 2 and 5 (sensitivity pattern b) and also in patients 6 and 7 (sensitivity patterns $\mathrm{g}$ and $\mathrm{h}$ ). However, the similarity of these strains was only confirmed in patients 2 and 5 by PFGE. Indeed, these two patients were present on the bone marrow transplant unit during the same time period, suggesting a common source of CoNS.

In conclusion, results from the current study highlight that genotypic typing techniques such as PFGE are more discriminatory than phenotypic typing methods for the identification and characterization of CoNS. Basing the 


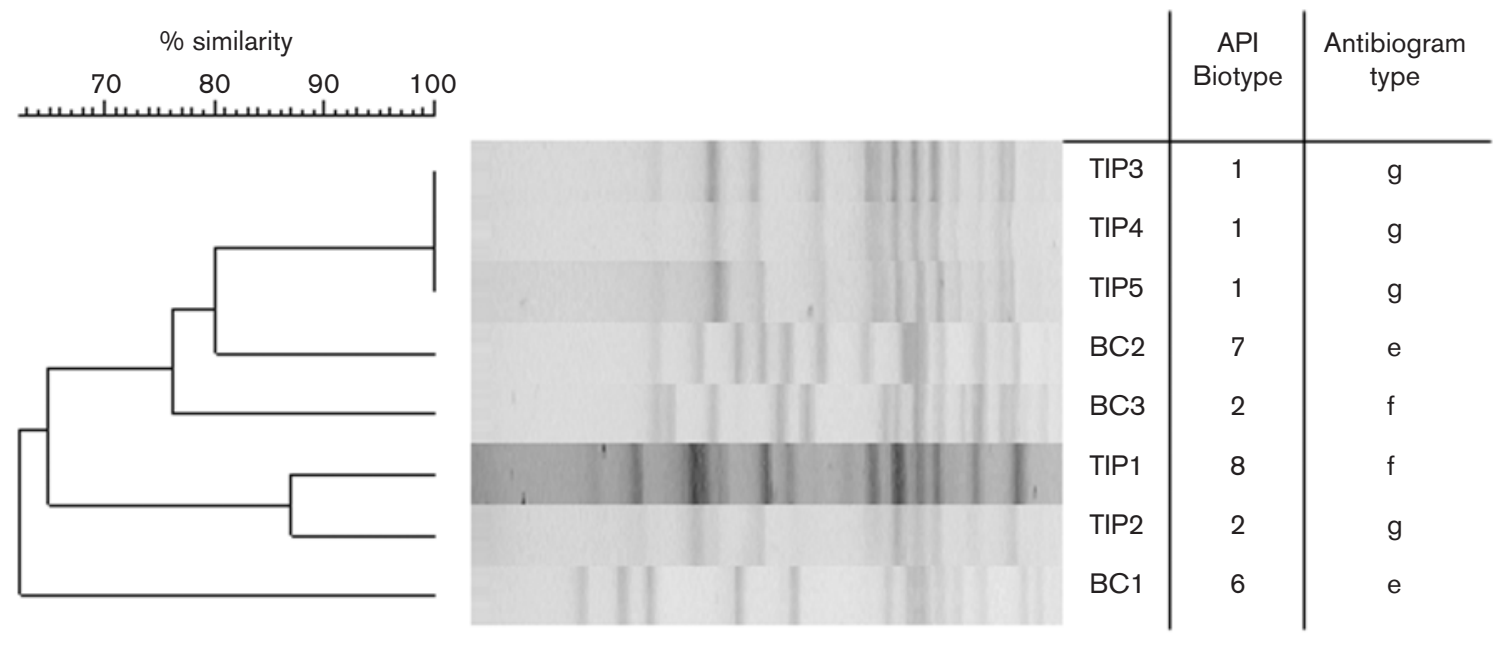

Fig. 2. Dendrogram of Smal macrorestriction profiles, biotype and antibiogram types of eight isolates of CoNS recovered from patient 8. API biotype profiles: $1=166012200 ; 2=366012200 ; 6=266314200 ; 7=166010200 ; 8=363002200$. Antibiogram typing profiles: e, resistant to oxacillin, erythromycin, trimethoprim, fusidin, mupirocin, gentamicin and clindamycin and sensitive to rifampicin and tetracycline; $f$, sensitive to oxacillin, erythromycin, trimethoprim, fusidin, rifampicin, mupirocin, tetracycline, gentamicin and clindamycin; g, resistant to oxacillin, erythromycin, trimethoprim, fusidin, gentamicin and clindamycin and sensitive to rifampicin, tetracycline and mupirocin. Using biotyping and antibiogram typing it is possible to see that specific isolates of CoNS recovered from the tip were indistinguishable from particular isolates recovered from the blood cultures; i.e. using antibiogram typing blood culture isolate 3 was indistinguishable from catheter tip isolate 1. Using PFGE no catheter tip isolate was indistinguishable from any blood culture isolate, discrediting the diagnosis of CR-BSI. Also, using all three typing techniques it was demonstrated that the catheter tip and blood cultures identified as being pure by the absence of differences in colonial morphology following $24 \mathrm{~h}$ incubation actually contained more than one strain of CoNS.

diagnosis of CR-BSI on phenotypic techniques alone may be misleading and needs to be considered by clinicians in interpreting blood and catheter cultures. Multiple colonies of CoNS should ideally be selected and subcultured for individual characterization to facilitate the diagnosis of CRBSI, especially since several strains may be present.

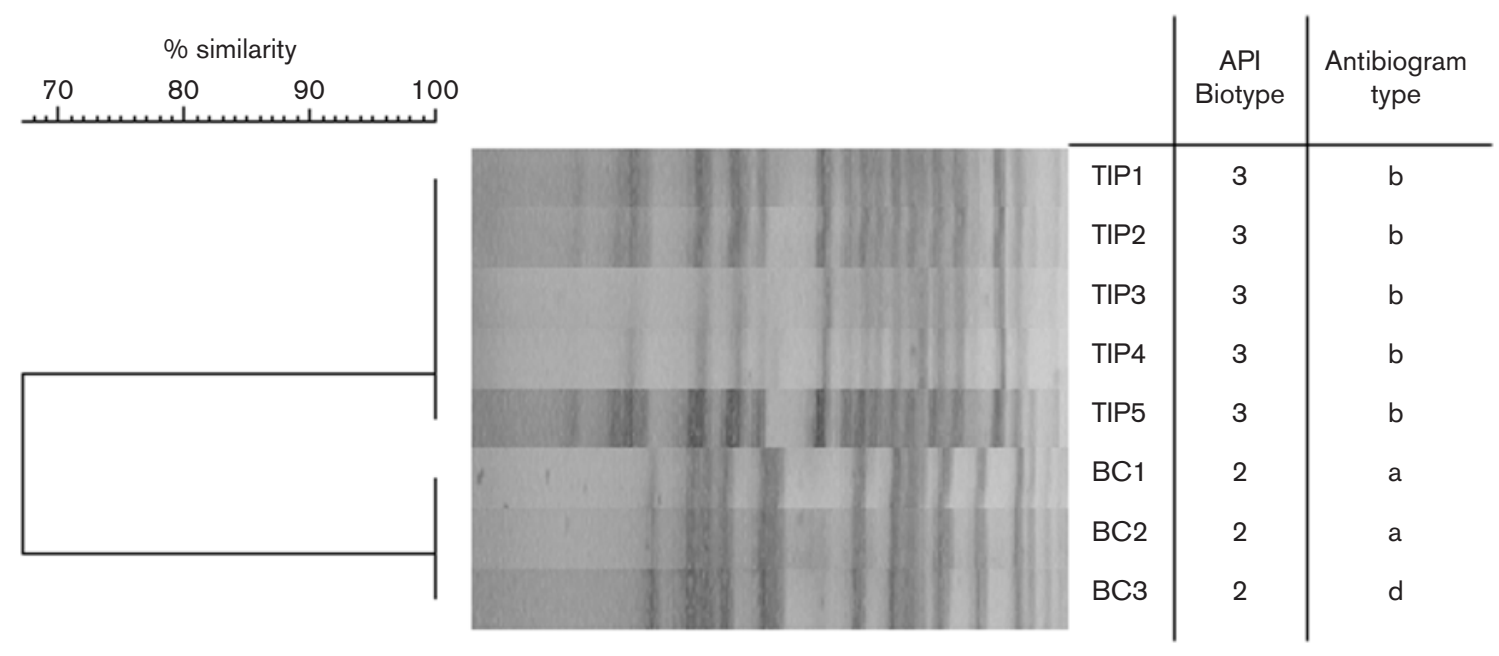

Fig. 3. Dendrogram of Smal macrorestriction profiles, biotype and antibiogram types of eight isolates of CoNS recovered from patient 4. API biotype profiles: $2=366012200 ; 3=2661$ 14610. Antibiogram typing profiles: a, resistant to oxacillin, erythromycin and gentamicin and sensitive to trimethoprim, fusidin, rifampicin, clindamycin, mupirocin and tetracycline; $b$, resistant to oxacillin, erythromycin, trimethoprim, fusidin, rifampicin, gentamicin, clindamycin and tetracycline and sensitive to mupirocin; $d$, resistant to oxacillin, trimethoprim and gentamicin and sensitive to erythromycin, fusidin, rifampicin, mupirocin, tetracycline and clindamycin. Using all three typing techniques it is possible to see that no blood culture isolates were indistinguishable from catheter tip isolates, discrediting the diagnosis of CR-BSI. 
Extending the incubation period of the initial blood and catheter cultures to $72 \mathrm{~h}$ may enable several colony types to be more readily identified; however, it is evident that rapid, cost-effective methods for genotyping CoNS such as those described recently need to be more readily available (Casey et al., 2005).

\section{REFERENCES}

Andrews, J. M. (2001). BSAC Working Party On Susceptibility Testing. BSAC standardized disc susceptibility testing method. J Antimicrob Chemother 48 (Suppl. 1), 43-57.

Casey, A. L., Worthington, T., Caddick, J. M., Hilton, A. C., Lambert, P. A. \& Elliott, T. S. J. (2005). RAPD for the typing of coagulasenegative staphylococci implicated in catheter-related bloodstream infection. J Infect 52, 282-289.

Chandran, A. U. \& Rennie, R. (2005). Routine antimicrobial susceptibility testing of coagulase-negative staphylococci isolated from blood cultures: is it necessary? Clin Microbiol Infect 11, 1037-1040.

Kloos, W. E. \& Bannerman, T. L. (1994). Update on clinical significance of coagulase-negative staphylococci. Clin Microbiol Rev 7, 117-140.
Maki, D. G., Weise, C. E. \& Sarafin, H. W. (1977). A semiquantitative culture method for identifying intravenous-catheter-related infection. N Engl J Med 296, 1305-1309.

O'Grady, N. P., Alexander, M., Dellinger, E. P., Gerberding, J. L., Heard, S. O., Maki, D. G., Masur, H., McCormick, R. D., Mermel, L. A. \& other authors (2002). Guidelines for the prevention of intravascular catheter-related infections. Pediatrics 110, e51.

Tenover, F. C., Arbeit, R. D., Archer, G., Biddle, J., Byrne, S., Goering, R., Hancock, G., Hebert, G. A., Hill, B. \& other authors (1994). Comparison of traditional and molecular methods of typing isolates of Staphylococcus aureus. J Clin Microbiol 32, 407-415.

Tenover, F. C., Arbeit, R. D., Goering, R. V., Mickelsen, P. A., Murray, B. E., Persing, D. H. \& Swaminathan, B. (1995). Interpreting chromosomal DNA restriction patterns produced by pulsed-field gel electrophoresis: criteria for bacterial strain typing. J Clin Microbiol 33, 2233-2239.

Toldos, C. M., Yague, G., Ortiz, G. \& Segovia, M. (1997). Assessment of multiple coagulase-negative staphylococci isolated in blood cultures using pulsed-field gel electrophoresis. Eur J Clin Microbiol Infect Dis 16, 581-586.

Viedma, D. G., Rabadan, P. M., Diaz, M., Cercenado, E. \& Bouza, E. (2000). Heterogeneous antimicrobial resistance patterns in polyclonal populations of coagulase-negative staphylococci isolated from catheters. J Clin Microbiol 38, 1359-1363.

Worthington, T. \& Elliott, T. S. J. (2005). Diagnosis of central venous catheter related infection in adult patients. J Infect 51, 267-280. 